\title{
Aspectos fitossociológicos e de crescimento de Commiphora leptophloeos no semiárido brasileiro
}

\author{
Renata Christina Souza Silva ${ }^{1}$, Rinaldo Luiz Caraciolo Ferreira ${ }^{1 *}$, José Antônio Aleixo da Silva1', Isabelle Maria Jacqueline Meunier ${ }^{1}$, \\ Rute Berger ${ }^{1}$
}

${ }^{1}$ Universidade Federal Rural de Pernambuco, Rua Dom Manoel de Medeiros, s/n, Dois Irmãos, CEP 52171-900, Recife, PE, Brasil

*Autor correspondente:

rinaldo.ferreira@ufrpe.br

Termos para indexação:

Dominância

Incremento periódico

Regeneração natural

Index terms:

Dominance

Periodic increment

Natural regeneration

Histórico do artigo:

Recebido em 12/04/2016

Aprovado em 23/03/2017

Publicado em 31/03/2017

doi: 10.4336/2017.pfb.37.89.1224

\begin{abstract}
Resumo - Objetivou-se analisar aspectos fitossociológicos e de crescimento de Commiphora leptophloeos (Mart.) J. B. Gillett. no semiárido brasileiro. A espécie foi estudada por meio de pesquisa bibliográfica e dados de 40 parcelas permanentes em área de caatinga, instaladas em 2008 e remensuradas em 2013, estimando-se densidade relativa (DR), frequência relativa (FR), dominância relativa (DoR), valor de importância (VI), regeneração natural $(\mathrm{RN})$, crescimento em área basal $(\mathrm{CB})$ e incremento periódico anual (IPA). A espécie apresentou DR de 0,04 a 12,82\%, FR de 0,10 a 9,53 \%, DoR de 0,01 a $19,58 \%$, VI de 0,14 a $10,87 \%$ e RN de 0,83 a $2,34 \%$. O CB foi negativo, quando considerada a mortalidade. O IPA foi de 0,00 a $0,25 \mathrm{~cm} \mathrm{ano}^{-1}$. A espécie se destaca por sua DoR, no entanto, apresenta baixas DR, FR e RN. Apresenta crescimento lento, que aliado a problemas de $\mathrm{RN}$ e sua extração sem manejo pode restringir a sua continuidade na vegetação.
\end{abstract}

\section{Phytosociological aspects and growth of Commiphora leptophloeos in Brazilian Semiarid}

\begin{abstract}
We aimed to analyze phytosociological aspects and growth of Commiphora leptophloeos (Mart.) J. B. Gillett in Brazilian semiarid region. It was performed literature review and processed data from 40 permanent plots. They were installed in a Caatinga area, measured in 2008 and remeasured in 2013 to estimate relative density (DR), relative frequency (FR), relative dominance (DoR), importance value (VI), natural regeneration $(\mathrm{RN})$, growth in basal area $(\mathrm{CB})$ and periodic annual increment (IPA). The specie showed DR from 2.58 to $12.82 \%$, FR from 0.10 to $9.53 \%$, DoR from 0.01 to $4.21 \%$, VI from 0.14 to $10.87 \%$ and $\mathrm{RN}$ from 0.83 to $2.34 \%$. CB was negative when mortality was considered. IPA varied from 0.00 to $0.25 \mathrm{~cm} \mathrm{yr}^{-1}$. The species has excelled due to its DoR, however, it presents low DR, FR, and RN. The species has slow growth, which combined with RN problems and its use without management may restrict its continuity in vegetation.
\end{abstract}

\section{Introdução}

A dependência social e econômica de comunidades rurais da região Nordeste do Brasil em relação aos recursos florestais da Caatinga está associada ao fornecimento de produtos madeireiros e não madeireiros que essa vegetação proporciona. Dentre esses produtos, o madeireiro é considerado o mais importante para as comunidades da região.

A exploração madeireira na região é, na maioria dos casos, realizada de forma extrativista. Esse extrativismo tem sido intensivo e, por isso, preocupante por gerar impactos sobre as espécies vegetais, promovendo 
o desflorestamento de áreas naturais e a perda de diversidade biológica (Ramos, 2007).

Segundo Sampaio \& Gamarra-Rojas (2002), aproximadamente $80 \%$ da madeira extraída da Caatinga é consumida como fonte de energia (lenha e carvão), representando a maior pressão extrativista na região Nordeste. Figueirôa et al. (2005) afirmaram que o destaque dado à atividade de produção de lenha e carvão, por muitas vezes, limita e oculta o verdadeiro potencial da região para a produção de produtos mais nobres e, consequentemente, de maior valor e retorno econômico. No entanto, para outros fins, a utilização da madeira é limitada, devido à essa vegetação ter poucas árvores com fuste adequado para produção de tábuas, linhas, vigas, postes, dentre outros (Giulietti et al., 2002).

Uma espécie que se destaca quanto aos seus diversos usos madeireiros e não madeireiros é Commiphora leptophloeos (Mart.) J._B.Gillett, conhecida popularmente como imburana-de-cambão. Estudos etnobotânicos apontaram o conhecimento tradicional de diferentes categorias de uso dados à espécie por comunidades locais do Semiárido, destacando seu emprego em construções domésticas e rurais, como forragem e fitoterápico, além do uso tecnológico e como combustível (Ferraz et al., 2006; Lucena et al., 2012), bem como a confecção de peças artesanais esculpidas. Vale ressaltar que esta espécie brasileira não é endêmica da caatinga (Gillett, 1980), sendo, por exemplo, encontrada também em Goiás (Moreira, 1995; Silva \& Scariot, 2004). Ainda assim, estudos científicos ainda são incipientes quanto à sua ecologia e seus produtos madeireiros e não madeireiros.

Logo, a necessidade de madeira de C. leptophloeos pela população rural, destacando-se especialmente a confecção de peças artesanais esculpidas, que demanda toras de $30 \mathrm{~cm}$ de circunferência a 1,30 m do solo, leva a perguntas ainda com poucas respostas: que padrão fitossociológico tem a espécie conforme o local de ocorrência no semiárido? Qual o seu crescimento?

O objetivo deste trabalho foi analisar os aspectos fitossociológicos e de crescimento de Commiphora leptophloeos no Semiárido brasileiro.

\section{Material e métodos}

Commiphora leptophloeos (Mart.) J. B. Gillett, com sinonímia botânica de Bursera leptophloeos (Mart.) Engl. e Icica leptophloeos Mart., é uma espécie pertencente à família Burseraceae, com distribuição ampla e descontínua no Brasil (biomas Mata Atlântica, Caatinga e Cerrado). Recebe diversos nomes populares, dentre os quais imburana-de-cambão, falsa-imburana, imburana-de-abelha, imburana-vermelha, imburanabrava, imburana-de-espinho, amburana e umburana (Giulietti et al., 2002; Carvalho, 2008; Maia, 2012).

A imburana-de-cambão é uma árvore resinosa, de comportamento decíduo, heliófita, pioneira e xerófita. Seus indivíduos medem entre 6 e $9 \mathrm{~m}$ de altura e até $60 \mathrm{~cm}$ de diâmetro a 1,30 m do solo (DAP) (Carvalho, 2008).

A distribuição dessa espécie, em áreas de caatinga, pode ser observada em estudos realizados na Paraíba (Lacerda et al., 2005; Fabricante \& Andrade, 2007; Oliveira et al., 2009; Araújo et al., 2012a, 2012b; Guedes et al., 2012; Souza, 2012), em Pernambuco (Rodal et al., 1999; Alcoforado Filho et al., 2003; Gomes et al., 2006; Pinheiro et al., 2010; Lima \& Rodal, 2010; Barbosa et al., 2012; Ferraz et al., 2012, 2014), no Ceará (Araújo et al., 1998; Lima et al., 2009), no Piauí (Mendes \& Castro, 2010), no Rio Grande do Norte (Cestaro \& Soares, 2004; Amorim et al., 2005; Vieira Júnior, 2014), na Bahia (Lima \& Lima, 1998), em Sergipe (Ferraz et al., 2013) e em Minas Gerais (Santos et al., 2008).

\section{Aspectos fitossociológicos e crescimento de Commiphora leptophloeos}

Foram utilizados dados de 40 parcelas permanentes, com dimensões de $20 \mathrm{~m}$ x $20 \mathrm{~m}$ cada, instaladas em 2008 (Alves Júnior, 2010), para mensuração de indivíduos lenhosos adultos (circunferência a 1,30 m do solo - CAP $\geq 6 \mathrm{~cm}$ ), em área de Savana-Estépica Arborizada - Caatinga (IBGE, 2012) em Floresta, PE, e remensuradas em 2013, visando estimar o crescimento e parâmetros fitossociológicos de todas as espécies. Porém, no presente estudo, evidenciou-se resultados apenas de C. leptophloeos.

As estimativas de densidade, frequência, dominância e valor de importância foram obtidas por meio das Equações 1, a 4, respectivamente.

$$
\mathrm{DR}=\left(\frac{n}{\sum_{i=1}^{p} n_{i}}\right) \cdot 100
$$




$$
\begin{aligned}
& \mathrm{FR}=\left(\frac{\mathrm{FA}}{\sum_{i=1}^{p} F A_{i}}\right) \cdot 100 \\
& \mathrm{DoR}=\left(\frac{G}{\sum_{i=1}^{p} G_{i}}\right) \cdot 100 \\
& \mathrm{VI}=\left(\frac{\mathrm{DR}^{2} \mathrm{FR}+\mathrm{DoR}^{p}}{\sum_{i=1}^{p}\left(\mathrm{DR}_{i}+\mathrm{FR}_{i}+\mathrm{DoR}_{i}\right)}\right) \cdot 100
\end{aligned}
$$

Em que: DR = densidade relativa da imburana-decambão (\%); $\mathrm{n}=$ número de indivíduos amostrados de imburana-de-cambão $\left(\mathrm{n} \mathrm{ha}^{-1}\right) ; \mathrm{n}_{\mathrm{i}}=$ número de indivíduos da i-ésima espécie amostrada $-\mathrm{n}_{\mathrm{i}} \mathrm{ha}^{-1}(\mathrm{i}=1,2, \ldots, \mathrm{p})$; $\mathrm{p}=$ número de espécies amostradas no levantamento; $\mathrm{FR}=$ frequência relativa de imburana-de-cambão (\%); $\mathrm{FA}=$ frequência absoluta de imburana-de-cambão (100 $\mathrm{x}$ (número de unidades amostrais em que a imburanade-cambão ocorre/número total de unidades amostrais); $\mathrm{FA}_{\mathrm{i}}=$ frequência absoluta da i-ésima espécie amostrada (\%); DoR = dominância relativa (\%); G = área basal da imburana-de-cambão $\left(\mathrm{m}^{2} \mathrm{ha}^{-1}\right)$; Gi = área basal da i-ésima espécie amostrada $\left(\mathrm{m}^{2} \mathrm{ha}^{-1}\right) ; \mathrm{VI}=$ valor de importância relativo (\%); $\mathrm{DR}_{\mathrm{i}}=$ densidade relativa (\%) da i-ésima espécie amostrada; $\mathrm{FRi}=$ frequência relativa (\%) da i-ésima espécie amostrada; $\mathrm{DoR}_{\mathrm{i}}=$ dominância relativa (\%) da i-ésima espécie amostrada.

Para o estudo da regeneração natural, foram utilizadas 40 subparcelas, com dimensões de $5 \mathrm{~m} \mathrm{x} 5 \mathrm{~m}$ cada, demarcadas no vértice superior direito (sentido sul-norte) de parcelas permanentes para os levantamentos dos indivíduos adultos. Em cada parcela, foram mensurados os indivíduos lenhosos de todas as espécies em estádio de regeneração, classificados em quatro classes de tamanho: classe I $=20-50 \mathrm{~cm}$; classe II $=51-100 \mathrm{~cm}$; classe III $=101-150 \mathrm{~cm}$; classe IV = maior que $151 \mathrm{~cm}$ de altura e menores que $6 \mathrm{~cm}$ de CAP. A regeneração natural de C. leptophloeos no período de 2008 a 2013 foi calculada utilizando-se a Equação 5 de.

$$
\mathrm{RNR}=\frac{\sum_{i=1}^{4}\left(\mathrm{DRC}_{i}+\mathrm{FRC}_{i}\right)}{8}
$$

Em que: $\mathrm{DRC}_{\mathrm{i}}=$ densidade relativa na i-ésima classe de regeneração; $\mathrm{FRC}_{\mathrm{i}}=$ frequência relativa na i-ésima classe de regeneração; RNR = regeneração natural relativa $(\%)$.

As estimativas de crescimento de C. leptophloeos foram obtidas por meio da metodologia de Beers (1962) modificada por Ferreira et al. (2014), empregando-se as Equações 6 a 9 .

$$
\begin{aligned}
& \mathrm{Cb}_{\mathrm{i}}=\mathrm{G} 2+\mathrm{M}+\mathrm{MF}+\mathrm{C}+\mathrm{CF}-\mathrm{G} 1 \\
& \mathrm{Cb}=\mathrm{G} 2+\mathrm{M}+\mathrm{MF}+\mathrm{C}+\mathrm{CF}-\mathrm{GI}-\mathrm{G} 1 \\
& \mathrm{Cl}_{\mathrm{i}}=\mathrm{G} 2+\mathrm{C}+\mathrm{CF}-\mathrm{G} 1 \\
& \mathrm{Cl}=\mathrm{G} 2+\mathrm{C}+\mathrm{CF}-\mathrm{GI}-\mathrm{G} 1
\end{aligned}
$$

Em que: $\mathrm{Cb}_{\mathrm{i}}=$ crescimento bruto incluindo-se ingressos $\left(\mathrm{m}^{2} \mathrm{ha}^{-1}\right) ; \mathrm{Cb}=$ crescimento bruto excluindo-se ingressos $\left(\mathrm{m}^{2} \mathrm{ha}^{-1}\right): \mathrm{Cl}_{\mathrm{i}}=$ crescimento líquido incluindose ingressos $\left(\mathrm{m}^{2} \mathrm{ha}^{-1}\right) ; \mathrm{Cl}=$ crescimento líquido excluindo-se ingressos $\left(\mathrm{m}^{2} \mathrm{ha}^{-1}\right)$; G1 = área basal de indivíduos vivos no início do período de mensuração $\left(\mathrm{m}^{2} \mathrm{ha}^{-1}\right) ; \mathrm{G} 2$ = área basal de indivíduos vivos no final do período de mensuração $\left(\mathrm{m}^{2} \mathrm{ha}^{-1}\right) ; \mathrm{M}=$ área basal de indivíduos mortos durante o período $\left(\mathrm{m}^{2} \mathrm{ha}^{-1}\right)$; $\mathrm{MF}=$ área basal de fustes mortos durante o período $\left(\mathrm{m}^{2} \mathrm{ha}^{-1}\right)$; $\mathrm{C}=$ área basal de indivíduos colhidos durante o período $\left(\mathrm{m}^{2} \mathrm{ha}^{-1}\right) ; \mathrm{CF}=$ área basal de fustes colhidos durante o período $\left(\mathrm{m}^{2} \mathrm{ha}^{-1}\right)$; $\mathrm{GI}=$ área basal de indivíduos ingressos no período $\left(\mathrm{m}^{2} \mathrm{ha}^{-1}\right)$ e $\mathrm{GF}=$ área basal de fustes ingressos no período $\left(\mathrm{m}^{2} \mathrm{ha}^{-1}\right)$.

Os incrementos periódicos anuais em área basal (IPA, $\mathrm{m}^{2} \mathrm{ha}^{-1}$ ano $^{-1}$ ) de C. leptophloeos foram obtidos considerando-se como a razão entre $\mathrm{Cb}_{\mathrm{i}}, \mathrm{Cb}, \mathrm{Cl}_{\mathrm{i}}$ e $\mathrm{Cl}$, respectivamente, e o intervalo entre ocasiões de mensuração (5 anos).

A partir da estimativa de $\mathrm{Cb}$, foi estimado o incremento periódico médio anual em diâmetro (Equação 10).

$$
\overline{\mathrm{IPA}}_{d}=\frac{1}{n \cdot a} \sqrt{\frac{40000 \cdot C_{b}}{\pi}}
$$

Em que: $\overline{\mathrm{IPA}}_{d}=$ incremento médio anual em diâmetro $\left(\mathrm{cm} \mathrm{ano}^{-1}\right) ; \mathrm{Cb}=$ crescimento bruto, excluindose ingressos $\left(\mathrm{m}^{2}\right.$ ha $\left.{ }^{-1}\right) ; \mathrm{n}=$ número de indivíduos $\left(\mathrm{n} \mathrm{ha}^{-1}\right)$; $\mathrm{a}=$ intervalo entre ocasiões de mensuração ( 5 anos). 


\section{Resultados e discussão}

Os parâmetros fitossociológicos para Commiphora leptophloeos em diferentes locais do Semiárido brasileiro estão apresentados na Tabela 1. A maioria dos trabalhos foi desenvolvida na Paraíba (46,12\%) e em Pernambuco $(23,07 \%)$, com dados restritos a estrutura horizontal da espécie (densidade, frequência, dominância e valor de importância). Os maiores valores de densidade relativa foram encontrados por Guedes et al. (2012), com 10,79\% e Guerra et al. (2014), com 12,82\%, na Paraíba e no Rio Grande do Norte, respectivamente. Nos demais trabalhos, $C$. leptophloeos apresentou resultados semelhantes, com densidade relativa variando entre 0,04 e $4,97 \%$, frequência relativa, entre 0,04 e 12,82\%, enquanto a dominância relativa variou de 0,01 a 19,58\%, resultando em amplitude de valor de importância de 0,14 a $10,87 \%$.

Vale ressaltar que as comparações devem ser realizadas com parcimônia, uma vez que as áreas contam com diferentes históricos de uso e de perturbação da vegetação, apesar de que a maioria dos trabalhos foi realizada em áreas com cerca de 25 anos sem maiores perturbações por exploração de madeira ou cultivo agrícola, mas com registro de pastejo de bovinos e/ou caprinos.

De uma forma geral, C. leptophloeos apresentou baixos valores de densidade nos levantamentos fitossociológicos. Por outro lado, obteve destaque quanto à dominância relativa, fato explicável pelo seu porte arbóreo. Vale ressaltar que o critério ou nível de inclusão não é igual para todos os trabalhos listados na Tabela 1 , logo, a comparação entre eles deve ser realizada com parcimônia.

Foram encontrados quatro trabalhos sobre a regeneração natural (RN) da espécie, sendo três realizados na Paraíba (Alves et al., $2010-\mathrm{RN}=1,27 \%$; Andrade et al., $2009-\mathrm{RN}=0,83 \%$; Pereira et al., 2001 - $\mathrm{RN}=1,18 ; 1,02$ e 2,34\%); um em Pernambuco (Alves Júnior et al., 2013 - 0,87\%) e um no Rio Grande do Norte (Vieira Júnior, 2014 - 1,18\%). No presente trabalho, a regeneração natural da imburana foi estimada em 1,45\%, o que corrobora com os demais trabalhos, constatando que a espécie apresenta problemas para se estabelecer devido aos baixos valores de regeneração natural (entre $0,83$ e $2,34 \%)$.

Pesq. flor. bras., Colombo, v. 37, n. 89, p. 11-18, jan./mar. 2017
Nas 40 parcelas levantadas em 2008, a espécie apareceu em apenas 15, com variação de 1 a 6 fustes por indivíduo e média de 1,72. A densidade observada foi, aproximadamente, 18 ind ha $\mathrm{h}^{-1} \mathrm{e} 31$ fustes $\mathrm{ha}^{-1}$. Em 2013, houve registro da espécie em apenas 13 parcelas, com igual variação no número de fustes por indivíduo (média $=2,00$ ).

Quando comparados os parâmetros fitossociológicos aos dados encontrados por Alves Júnior (2010), foi observado aumento da densidade relativa $(1,02 \%$, em 2008 e 2,58\%, em 2013), estabilidade na frequência relativa $(4,13 \%$ em 2008 e $4,04 \%$ em 2013) e nos valores de dominância relativa $(4,22 \%$ em 2008 e 4,21\%, em 2013). No mesmo local, Pimentel (2011) observou redução da densidade relativa da imburana entre os anos de 2008 e 2011 com 0,36 e 0,25\%, respectivamente, estabilidade da frequência relativa $(2,04 \%$ em 2008 e em 2011) e aumento da dominância relativa $(0,12 \%$ em 2008 e 5,13\% em 2011). Barreto (2013), na mesma área de caatinga e em outra área próxima, encontrou estabilidade dos valores relativos de densidade, frequência e dominância entre 2008 e $2012(D R=0,30$ e $0,29, \mathrm{FR}=2,30$ nos dois anos e $\mathrm{DoR}=0,10$ e 0,12 ).

$\mathrm{Na}$ análise da regeneração natural de todas as espécies, foi observado um único indivíduo de C. leptopholeos pertencente à classe III (101 a $150 \mathrm{~cm}$ de altura). Alves Júnior et al. (2013), em levantamento realizado em 2008, nas mesmas parcelas deste trabalho, também observaram apenas um indivíduo regenerante na classe III. Segundo os autores, esse comportamento demonstra que a espécie não dispõe de recrutamentos contínuos. Pimentel (2011), em levantamento de área de caatinga no município de Floresta, PE, não encontrou indivíduos regenerantes de C. leptophloeos em nenhuma das ocasiões de monitoramento (2008, 2009, 2010 e 2011), corroborando com a argumentação de que a espécie apresenta restrições para sua regeneração.

Houve crescimento negativo em área basal quando não se considerou a mortalidade (Tabela 2), resultado contrário ao de Pimentel (2011) que observou crescimentos líquidos incluindo ingresso $-\mathrm{Cl}_{\mathrm{i}}(0,1184$ $\left.\mathrm{m}^{2} \mathrm{ha}^{-1}\right)$ e excluindo ingresso - $\mathrm{Cl}\left(0,0010 \mathrm{~m}^{2} \mathrm{ha}^{-1}\right)$, na mesma área do presente estudo, no período 2008-2011. A diferença entre os estudos pode ser atribuída à morte do indivíduo de maior porte $(\mathrm{CAP}=95,5 \mathrm{~cm})$. Barreto (2013) não observou mortalidade e registrou $\mathrm{Cl}_{\mathrm{i}}(0,0059$ $\left.\mathrm{m}^{2} \mathrm{ha}^{-1}\right)$ e $\mathrm{Cl}\left(0,0045 \mathrm{~m}^{2} \mathrm{ha}^{-1}\right)$, na mesma área de caatinga e em outra área próxima, no período entre 2008 e 2012. 
Tabela 1. Estimativa de parâmetros fitossociológicos para Commiphora leptophloeos em diferentes locais do semiárido brasileiro.

\begin{tabular}{|c|c|c|c|c|c|c|}
\hline \multirow{2}{*}{ Autor (es) } & \multirow{2}{*}{ Local } & \multirow{2}{*}{ Coordenadas } & DR & FR & DoR & VI \\
\hline & & & \multicolumn{4}{|c|}{$(\%)$} \\
\hline Presente trabalho & Floresta, PE & $8^{\circ} 30^{\prime} 37^{\prime \prime}$ S e $37^{\circ} 59^{\prime} 07^{\prime \prime} \mathrm{W}$ & 2,58 & 4,04 & 4,21 & 3,61 \\
\hline \multirow{2}{*}{ Ferraz et al. (2014) } & \multirow{2}{*}{ Floresta, PE } & \multirow{2}{*}{$8^{\circ} 33220,93 \mathrm{~S}$ e $37^{\circ} 56227,43 \mathrm{~W}$} & 0,24 & 1,51 & 0,14 & 0,63 \\
\hline & & & 0,89 & 3,50 & 11,33 & 5,24 \\
\hline Guerra et al. (2014) & Apodi, RN & $05^{\circ} 32^{\prime \prime S}$ e $37^{\circ} 52^{\prime \prime} \mathrm{W}$ & 12,82 & 7,00 & 6,83 & 8,86 \\
\hline Vieira Júnior (2014) & Caicó, RN & $05^{\circ} 32^{\prime} 33,5^{\prime \prime}$ S e $37^{\circ} 06^{\prime \prime} 1,16^{\prime \prime} \mathrm{W}$ & 1,19 & 4,48 & 1,65 & 2,44 \\
\hline Cunha et al. (2013) & Maturéia e Mãe d'Água, PB & $\begin{array}{l}06^{\circ} 02^{\prime} 12^{\prime \prime} \text { e } 08^{\circ} 19^{\prime} 18^{\prime \prime} \mathrm{S} \mathrm{e} \\
34^{\circ} 45^{\prime} 12^{\prime \prime} \text { e } 38^{\circ} 45^{\prime} 45^{\prime \prime} \mathrm{W}\end{array}$ & 0,05 & 0,20 & 0,48 & 0,73 \\
\hline Ferraz et al. (2013) & Canindé do São Francisco, SE & $9^{\circ} 39^{\prime} 36^{\prime \prime} \mathrm{S}$ e $37^{\circ} 47^{\prime} 22^{\prime \prime} \mathrm{W}$ & 1,66 & 8,52 & 5,66 & 5,28 \\
\hline Marangon et al. (2013) & Floresta, PE & $8^{\circ} 36^{\prime} 04^{\prime \prime}$ S e $38^{\circ} 34^{\prime} 07^{\prime \prime} \mathrm{W}$ & 0,82 & 3,52 & 10,76 & 5,03 \\
\hline Araújo et al. (2012a) & Santa Luzia, PB & $6^{\circ} 52^{\prime} 27^{\prime \prime}$ S e $36^{\circ} 56^{\prime} 00^{\prime \prime} \mathrm{W}$ & 1,14 & - & 7,68 & \\
\hline Araujo et al. (2012b) & São João do Cariri, PB & $7^{\circ} 23^{\prime} 30^{\prime \prime}$ S e $36^{\circ} 31^{\prime} 59^{\prime \prime} \mathrm{W}$ & 0,05 & 0,56 & 0,15 & \\
\hline Barbosa et al. (2012) & Arcoverde, $\mathrm{PE}$ & $8^{\circ} 01^{\prime} 03,6^{\prime \prime} \mathrm{S}$ e $34^{\circ} 56^{\prime} 44,1^{\prime \prime} \mathrm{W}$ & 0,07 & 0,35 & 0,01 & 0,14 \\
\hline Carvalho et al. (2012) & Barra de Santana, PB & $7^{\circ} 31^{\prime} 12^{\prime \prime}$ S e $35^{\circ} 59^{\prime} 59^{\prime \prime} \mathrm{W}$ & & & & 3,6 \\
\hline Guedes et al. (2012) & Santa Terezinha, PB & $7^{\circ} 1^{\prime}$ S e $37^{\circ} 24^{\prime} \mathrm{W}$ & 10,79 & 6,84 & 10,38 & 9,33 \\
\hline Pereira Júnior et al. (2012) & Monteiro, PB & $7^{\circ} 50^{\prime} 13,92^{\prime \prime}$ S e $37^{\circ} 11^{\prime} 29,76^{\prime \prime} \mathrm{W}$ & 0,26 & 0,99 & 0,28 & 0,51 \\
\hline Souza (2012) & São José dos Espinharas, PB & $\begin{array}{c}6^{\circ} 48^{\prime} 33,11^{\prime \prime}, 6^{\circ} 47^{\prime} 09,94 " \mathrm{~S}, \mathrm{e} \\
37^{\circ} 19^{\prime} 53,64^{\prime \prime}, 37^{\circ} 19^{\prime} 23,66^{\prime \prime} \mathrm{W}\end{array}$ & 0,41 & 2,61 & 0,76 & 1,26 \\
\hline Bessa \& Medeiros (2011) & Taboleiro Grande, RN & $5^{\circ} 55^{\prime} 48^{\prime \prime}$ S e $38^{\circ} 02^{\prime} 42^{\prime \prime} \mathrm{W}$ & 0,35 & 2,12 & 0,46 & 0,98 \\
\hline Alves Júnior (2010) & Floresta, PE & $8^{\circ} 30^{\prime} 37^{\prime \prime}$ S e $37^{\circ} 59^{\prime} 07^{\prime \prime} \mathrm{W}$ & 1,02 & 4,13 & 4,22 & 3,13 \\
\hline Alves et al. (2010) & Pombal, PB & $6042^{\prime} 10^{\prime \prime} \mathrm{S}$ e $37045^{\prime} 15^{\prime \prime} \mathrm{W}$ & 0,61 & 1,92 & & \\
\hline Dantas et al. (2010) & Pombal, PB & $6042^{\prime} 10^{\prime \prime} \mathrm{S}$ e $37045^{\prime} 15^{\prime \prime} \mathrm{W}$ & 0,11 & 0,94 & 0,29 & 1,34 \\
\hline Parente et al. (2010) & São João do Cariri, PB & $7^{\circ} 23^{\prime} 30^{\prime \prime}$ S e $36^{\circ} 31^{\prime} 59^{\prime \prime} \mathrm{W}$ & 0,05 & 0,56 & 0,15 & 0,26 \\
\hline Andrade et al. (2009) & Carnaúba dos Dantas, PB & Não informadas & 4,76 & 5,6 & 19,21 & 9,86 \\
\hline Lima (2006) & Crateús, CE & $5^{\circ} 05^{\prime}, 5^{\circ} 15^{\prime}$ S e $40^{\circ} 51^{\prime}, 41^{\circ} 00^{\prime} \mathrm{W}$ & 0,04 & 0,10 & 0,03 & 0,17 \\
\hline Oliveira et al. (2009) & Caturité, PB & Não informadas & 1,74 & 6,20 & 4,74 & 4,23 \\
\hline Oliveira et al. (2009) & Caturité, PB & Não informadas & 1,84 & 3,27 & 1,99 & 2,36 \\
\hline Oliveira et al. (2009) & Boqueirão, PB & Não informadas & 0,55 & 3,05 & 3,52 & 2,37 \\
\hline Oliveira et al. (2009) & Boqueirão, PB & Não informadas & 1,69 & 4,23 & 6,88 & 4,26 \\
\hline Rodal et al. (2008a) & Floresta e Betânia, PE & $\begin{array}{l}8^{\circ} 18^{\prime} 43^{\prime \prime} \mathrm{S} \mathrm{e} \\
38^{\circ} 11^{\prime} 45^{\prime \prime} \mathrm{W}\end{array}$ & 0,1 & 0,5 & 1,3 & 1,8 \\
\hline Rodal et al. (2008a) & Floresta-PE & $8^{\circ} 30^{\prime}-8^{\circ} 37^{\prime} \mathrm{S}$ e $38^{\circ} 00^{\prime}-38^{\circ} 17^{\prime} \mathrm{W}$ & 2,56 & 5,73 & 18,27 & 8,85 \\
\hline Rodal et al. (2008a) & Floresta-PE & $8^{\circ} 30^{\prime}-8^{\circ} 37^{\prime} \mathrm{S}$ e $38^{\circ} 00^{\prime}-38^{\circ} 17^{\prime} \mathrm{W}$ & 0,37 & 1,11 & 0,10 & 0,53 \\
\hline Rodal et al. (2008a) & Custódia-PE & $8^{\circ} 18^{\prime}$ 'S e $38^{\circ} 35^{\prime} \mathrm{W}$ & 0,21 & 0,66 & 2,80 & 1,22 \\
\hline Barbosa et al. (2007) & Fazenda Almas, PB & $7^{\circ} 28^{\prime} 15^{\prime \prime}$ S e $36^{\circ} 53^{\prime} 51^{\prime \prime} \mathrm{W}$ & 4,38 & 7,27 & 5,55 & 5,73 \\
\hline Barbosa et al. (2007) & São João do Cariri, PB & $7^{\circ} 23^{\prime} 48^{\prime \prime}$ S e $36^{\circ} 31^{\prime} 55^{\prime \prime} \mathrm{W}$ & 0,66 & 1,65 & 5,99 & 2,77 \\
\hline Fabricante \& Andrade (2007) & Santa Luzia, PB & $6^{\circ} 48^{\prime} 36,7^{\prime \prime}$ S e $36^{\circ} 57^{\prime} 38,8^{\prime \prime} \mathrm{W}$ & 2,08 & 7,39 & 3,46 & 4,31 \\
\hline Lira et al. (2007) & Assu, RN & $5^{\circ} 34^{\prime} 20^{\prime \prime} \mathrm{S}$ e $36^{\circ} 54^{\prime} 33^{\prime \prime} \mathrm{W}$ & 3,11 & 1,80 & 9,62 & 4,84 \\
\hline Moreira et al. (2007) & Carnaúbas, RN & $37^{\circ} 33^{\prime} 24^{\prime \prime} \mathrm{W}$ e $05^{\circ} 47^{\prime} 33^{\prime \prime} \mathrm{S}$ & 3,5 & 9,53 & 19,58 & 10,87 \\
\hline Queiroz et al. (2006) & Boqueirão, PB & Não informadas & 0,59 & 1,59 & & 3,07 \\
\hline Santana \& Souto (2006) & Serra Negra, RN & $6^{\circ} 34^{\prime} 00^{\prime \prime}-6^{\circ} 37^{\prime} 00^{\prime \prime}$ S e $37^{\circ} 15^{\prime} 37^{\circ} 16^{\prime} 00^{\prime \prime} \mathrm{W}$ & 3,47 & 6,10 & 4,17 & 4,58 \\
\hline Amorim et al. (2005) & Serra Negra do Norte, RN & $6^{\circ} 35^{\prime}-6^{\circ} 40^{\prime}$ S e $37^{\circ} 20^{\prime}-37^{\circ} 39^{\prime} \mathrm{W}$ & 3,47 & 0,30 & 0,02 & 1,26 \\
\hline Andrade et al. (2005) & São João do Cariri, PB & $7^{\circ} 24^{\prime \prime} 00 \mathrm{~S}$ e $36^{\circ} 32^{\prime \prime} 00 \mathrm{~W}$ & 0,18 & 1,49 & 1,72 & 1,13 \\
\hline Cestaro \& Soares (2004) & Macaíba, RN & $5^{\circ} 53^{\prime}$ 'S e $35^{\circ} 23^{\prime} \mathrm{W}$ & 1,48 & 1,79 & 1,69 & 1,65 \\
\hline Alcoforado Filho et al. (2003) & Caruaru, PE & $8^{\circ} 14^{\prime} 18^{\prime \prime}$ 'S e $35^{\circ} 55^{\prime} 20^{\prime \prime} \mathrm{W}$ & 1,53 & 3,29 & 5,88 & 3,57 \\
\hline Maracajá et al. (2003) & Serra do Mel, RN & $5^{\circ} 10^{\prime} 12^{\prime \prime}$ S e $37^{\circ} 01^{\prime} 46^{\prime \prime} \mathrm{W}$ & 1,44 & 6,90 & 0,12 & 2,82 \\
\hline Mendes (2003) & São José do Piauí, PI & $06^{\circ} 51^{\prime}$ S e $41^{\circ} 28^{\prime} \mathrm{W}$ & 0,36 & 0,56 & 0,52 & 0,48 \\
\hline Nascimento et al. (2003) & Petrolina, PE & $9^{\circ} 02^{\prime} \mathrm{S}$ e $40^{\circ} 14^{\prime} \mathrm{W}$ & 4,65 & 5,26 & 2,45 & 4,12 \\
\hline Pereira et al. (2002) & $\begin{array}{l}\text { Fronteira dos municípios de } \\
\text { Areia e Remígio, PB }\end{array}$ & $6^{\circ} 52^{\prime} 52^{\prime \prime} \mathrm{S}$ e $35^{\circ} 47^{\prime} 42^{\prime \prime} \mathrm{W}$ & 3,07 & 4,68 & 15,63 & 7,79 \\
\hline Lima \& Lima (1998) & Contendas do Sincorá, BA & $13^{\circ} 46^{\prime}, 14^{\circ} \mathrm{S}$ e $41^{\circ} 3^{\prime}, 41^{\circ} 10^{\prime} \mathrm{W}$ & 4,97 & 4,34 & 12,97 & 2,47 \\
\hline
\end{tabular}

$\mathrm{DR}=$ densidade relativa; $\mathrm{FR}=$ frequência relativa; $\mathrm{DoR}=$ dominância relativa; $\mathrm{VI}=$ valor de importância relativo. 
Tabela 2.Crescimento e incremento periódico de Commiphora leptophloeos em área basal $\left(\mathrm{m}^{2} \mathrm{ha}^{-1}\right)$ em área de Caatinga no período 2008-2013 em Floresta, PE.

\begin{tabular}{cccccc}
\hline \multicolumn{2}{c}{$\begin{array}{c}\text { Mortalidade } \\
\left(\text { número ha }{ }^{-1}\right)\end{array}$} & \multicolumn{2}{c}{$\begin{array}{c}\text { Crescimento bruto } \\
\left(\mathrm{m}^{2} \mathrm{ha}^{-1}\right)\end{array}$} & $\begin{array}{c}\text { Crescimento líquido } \\
\left(\mathrm{m}^{2} \mathrm{ha}^{-1}\right)\end{array}$ \\
\hline \multirow{3}{*}{ Fustes } & Indivíduos & $\begin{array}{c}\text { Incluindo } \\
\text { ingresso } \\
\left(\mathrm{Cb}_{\mathrm{i}}\right)\end{array}$ & $\begin{array}{c}\text { Excluindo } \\
\text { ingresso } \\
(\mathrm{Cb})\end{array}$ & $\begin{array}{c}\text { Incluindo } \\
\text { ingresso } \\
(\mathrm{Cli})\end{array}$ & $\begin{array}{c}\text { Excluindo } \\
\text { ingresso } \\
(\mathrm{Cl})\end{array}$ \\
\hline 0,000497 & 0,064739 & 0,014850 & 0,014850 & $-0,050387$ & $-0,050387$ \\
\hline & & \multicolumn{5}{c}{ Incremento periódico anual } \\
\cline { 2 - 7 } & & 0,002970 & 0,002970 & $-0,010080$ & $-0,010080$ \\
\hline
\end{tabular}

Obs.: não foram observados ingressos ou sinais de corte de indivíduos ou fustes.

No período entre 2008 e 2013 registrou-se mortalidade de nove indivíduos e de outro apenas um fuste. $\mathrm{O}$ incremento periódico anual (IPA) em diâmetro foi estimado em $0,14 \mathrm{~cm}$ ano ${ }^{-1}$, com variação de 0,00 a 0,25 $\mathrm{cm}$ ano $^{-1}$, sendo inferior aos observados por Pimentel (2011) e Barreto (2013), respectivamente, de 0,24 (estimado a partir do crescimento bruto, excluindo o ingresso) e $0,37 \mathrm{~cm} \mathrm{ano}^{-1}$.

O IPA em diâmetro também foi $50 \%$ inferior aos observados em outros estudos em florestas tropicais secas (Murphy \& Lugo, 1986; Swaine et al., 1990; Castro Marín et al., 2005). Vale ressaltar que as diferenças encontradas estão associadas à intensidade amostral, cuja representatividade é difícil de ser atendida em estudos de crescimento, ao qual estão associados diversos fatores como local, histórico de uso e de perturbações, sanidade, genética, clima, etc.

Esses resultados concordam com Carvalho (2008), indicando ser a espécie de crescimento lento, apesar dos poucos dados existentes em condições naturais e de plantio. Por outro lado, é importante ressaltar que esses resultados devem ser utilizados com parcimônia, especialmente devido aos poucos dados disponíveis sobre crescimento e produção da espécie, bem como aos diferentes históricos de uso e perturbação das áreas estudadas.

\section{Conclusões}

Commiphora leptopholeos tem se destacado fitossociologicamente devido à sua dominância relativa. No entanto, apresenta baixa densidade, frequência e regeneração natural.

A espécie apresenta crescimento lento que, aliado a problemas de regeneração natural e exploração extrativista, pode comprometer seriamente a continuidade de sua presença e regeneração nas formações vegetais naturais da Caatinga.

\section{Referências}

Alcoforado Filho, F. G. et al. Florística e fitossociologia de um remanescente de vegetação caducifólia espinhosa arbórea em Caruaru, Pernambuco. Acta Botanica Brasilica, v. 17, n. 2, p. 287 303, 2003. DOI: 10.1590/S0102-33062003000200011.

Alves Júnior, F. T. Estrutura, biomassa e volumetria de uma área de caatinga, Floresta-PE. 2010. 123 f. Tese (Doutorado em Ciências Florestais) - Universidade Federal Rural de Pernambuco, Recife.

Alves Júnior, F. T. et al. Regeneração natural de uma área de caatinga no Sertão Pernambucano, Nordeste do Brasil. Cerne, v. 19, n. 2, p. 229-235, 2013. DOI: 10.1590/S0104-77602013000200006.

Alves, L. S. et al. Regeneração natural em uma área de caatinga situada no município de Pombal-PB. Revista Verde, v. 5, n. 2, p. 152-168, 2010.

Amorim, I. L. et al. Flora e estrutura da vegetação arbustivo-arbórea de uma área de caatinga do Seridó, RN, Brasil. Acta Botânica Brasilica, v. 19, n. 3, p. 615-623, 2005. DOI: 10.1590/S010233062005000300023.

Andrade, L. A. et al. Análise da cobertura de duas fitofisionomias de caatinga, com diferentes históricos de uso, no Município de São João do Cariri, Estado da Paraíba. Cerne, v. 11, n. 3, p. 253-263, 2005.

Andrade, L. A. et al. Invasão biológica por Prosopis juliflora (Sw.) DC.: impactos sobre a diversidade e a estrutura do componente arbustivo-arbóreo da caatinga no Estado do Rio Grande do Norte, Brasil. Acta Botanica Brasilica, v. 23, n. 4, p. 935-943, 2009. DOI: 10.1590/S0102-33062009000400004.

Araújo, B. A. et al. Estrutura fitossociológica em uma área de caatinga no seridó paraibano. Revista Educação Agrícola Superior, v. 27, n. 1, p. 25-29, 2012a. DOI: 10.12722/0101-756X.v27n01a04.

Araújo, F. S. et al. Composição florística da vegetação de carrasco, Novo Oriente, CE. Revista Brasileira de Botânica, v. 21, n. 2, p. 1-19, 1998. DOI: 10.1590/S0100-84041998000200001.

Araújo, K. D. et al. Estrutura fitossociológica do estrato arbustivoarbóreo em áreas contíguas de Caatinga no Cariri Paraibano. Brazilian Geographical Journal: Geosciences and Humanities Research Medium, v. 3, n. 1, p. 155-169, 2012 b.

Barbosa, M. D. et al. Florística e fitossociologia de espécies arbóreas e arbustivas em uma área de caatinga em Arcoverde, PE, Brasil. Revista Árvore, v. 36, n. 5, p. 851-858, 2012. DOI: 10.1590/S010067622012000500007.

Barbosa, M. R. V. et al. Vegetação e flora no Cariri Paraibano. Oecologia Brasiliensis, v. 11, n. 3, p. 313-322, 2007.

Barreto, T. N. A. Dinâmica de espécies lenhosas em área de caatinga, Floresta-PE. 2013. 55 f. Dissertação (Mestrado em Ciências Florestais) - Universidade Federal Rural de Pernambuco, Recife. 
Beers, T. W. Components of forest growth. Journal of Forestry, v. 60, n. 4, p. 245-248, 1962.

Bessa, M. A. P. \& Medeiros, J. F. Levantamento florístico e fitossociológico em fragmentos de caatinga no município de Taboleiro Grande-RN. Revista Geotemas, v. 1, n. 2, p. 69-83, 2011.

Carvalho, E. C. D. et al. Ecological succession in two remnants of the Caatinga in the semi-arid tropics of Brazil. Revista Brasileira de Biociências, v. 10, n. 1, p. 13-19, 2012.

Carvalho, P. E. R. Espécies arbóreas brasileiras. Brasília, DF: Embrapa Informação Tecnológica; Colombo: Embrapa Florestas, 2008. 644 p.

Castro Marín, G. C. et al. Stand dynamics and basal area change in a tropical dry forest reserve in Nicaragua. Forest Ecology and Management, v. 208, n. 1, p. 63-75, 2005. DOI: 10.1016/j. foreco.2004.10.072.

Cestaro, L. A. \& Soares, J. J. Variações florística e estrutural e relações fitogeográficas de um fragmento de floresta decídua no Rio Grande do Norte, Brasil. Acta Botânica Brasilica, v. 18, n. 2, p. 203-218, 2004. DOI: 10.1590/S0102-33062004000200001.

Cunha, M. C. L. et al. Fitossociologia do estrato lenhoso de uma floresta estacional semidecidual montana na Paraíba, Brasil. Cerne, v. 19, n. 2, p. 271-280, 2013. DOI: 10.1590/S010477602013000200011 .

Dantas, J. G. et al. Estrutura do componente arbustivo/arbóreo de uma área de caatinga situada no município de Pombal-PB. Revista Verde, v. 5, n. 1, p. 134-142, 2010.

Fabricante, J. R. \& Andrade, L. A. Análise estrutural de um remanescente de caatinga no Seridó Paraibano. Oecologia Brasiliensis, v. 11, n. 3, p. 341-349, 2007.

Ferraz, J. S. F. et al. Estrutura do componente arbustivo-arbóreo da vegetação em duas áreas de caatinga, no município de Floresta, Pernambuco. Revista Árvore, v. 38, n. 6, p. 1055-1064, 2014. DOI: 10.1590/S0100-67622014000600010.

Ferraz, J. S. F. et al. Usos de especies leñosas de la caatinga del municipio de Floresta en Pernambuco, Brasil: conocimiento de los indios de la aldea Travessão do Ouro. Bosque, v. 33, n. 2, p. 183 190, 2012. DOI: 10.4067/S0717-92002012000200008.

Ferraz, J. S. F. et al. Valor de uso e estrutura da vegetação lenhosa às margens do riacho do Navio, Floresta, PE, Brasil. Acta Botanica Brasilica, v. 20, n. 1, p. 125-134, 2006. DOI: 10.1590/S010233062006000100012.

Ferraz, R. C. et al. Levantamento fitossociológico em área de caatinga no monumento natural Grota do Angico, Sergipe, Brasil. Revista Caatinga, v. 26, n. 3, p. 89-98, 2013.

Ferreira, R. L. C. et al. Components of growth for tropical dry deciduous forest, Brazil. In: ASA, CSSA \& SSSA INTERNATIONAL ANNUAL MEETING, 2014, Long Beach. Proceedings... Long Beach: ASA; CSSA; SSSA, 2014.

Figueirôa, J. M. et al. (Org.). Espécies da flora nordestina de importância econômica potencial. Recife: Editora Universitária; 2005. p. 101-133.
Gillett, J. B. Commiphora (Burseraceae) in South America and its relationship to Bursera. Kew Bulletin, v. 34, n. 3, p. 569-587, 1980. DOI: $10.2307 / 4109836$.

Giulietti, A. M. et al. Espécies endêmicas da caatinga. In: Sampaio, E. V. S. B. et al. (Ed.). Vegetação e flora das caatingas. Recife: APNE; CNIP, 2002. p. 103-108.

Gomes, A. P. S. et al. Florística e fitogeografia da vegetação arbustiva subcaducifólia da Chapada de São José, Buíque, PE, Brasil. Acta Botanica Brasilica, v. 20, n. 1, p. 37-48, 2006. DOI: 10.1590/S010233062006000100005.

Guedes, R. S. et al. Caracterização florístico-fitossociológica do componente lenhoso de um trecho de caatinga no Semiárido Paraibano. Revista Caatinga, v. 25, n. 2, p. 99-108, 2012.

Guerra, A. M. N. et al. Estudo fitossociológico em dois ambientes da caatinga localizada no assentamento Moacir Lucena, Apodi-RNBrasil. Revista Verde, v. 9, n. 1, p. 141-150, 2014.

IBGE. Manual técnico da vegetação brasileira. 2. ed. Rio de Janeiro, 2012. 274 p.

Lacerda, A.V. et al. Levantamento florístico do componente arbustivo-arbóreo da vegetação ciliar na bacia do rio Taperoá, PB, Brasil. Acta Botanica Brasilica, v. 19, n. 3, p. 647-656, 2005. DOI: 10.1590/S0102-33062005000300027.

Lima, A. L. A. \& Rodal, M. J. N. Phenology and wood density of plants growing in the semi-arid region of northeastern Brazil. Journal of Arid Environments, v. 74, n. 11, p. 1363-1373, 2010. DOI: 10.1016/j.jaridenv.2010.05.009.

Lima, J. R. et al. Composição florística da floresta estacional decídua montana de Serra das Almas, CE, Brasil. Acta Botanica Brasilica, v. 23, n. 3, p. 756-763, 2009. DOI: 10.1590/S010233062009000300015.

Lima, J. R. Florística e estrutura da floresta estacional decídua montana da RPPN Serra das Almas, Crateús, Ceará. 2006. 70 f. Dissertação (Mestrado em Botânica) - Universidade Federal Rural de Pernambuco, Recife.

Lima, P. C. F. \& Lima, J. L. S. Composição florística e fitossociologia de uma área de Caatinga em contendas do Sincorá, Bahia, Microrregião homogênea da chapada Diamantina. Acta Botanica Brasilica, v. 12, n. 3, supl. 1, p. 441-450, 1998. DOI: 10.1590/ S0102-33061998000400013.

Lira, R. B. et al. Estudo da composição florística arbóreo-arbustivo na Floresta Nacional de Açu no Semiárido do RN Brasil. Agropecuária Científica no Semi-Árido, v. 3, n. 1, p. 23-30, 2007.

Lucena, R. F. P. et al. Uso de recursos vegetais da caatinga em uma comunidade rural no Curimataú Paraibano (Nordeste do Brasil). Polibotánica, n. 34, p. 217-238, 2012.

Maia, G. N. Caatinga: árvores e arbustos e suas utilidades. 2. ed. Fortaleza: Printcolor, 2012. 413 p.

Maracajá, P. B. et al. Levantamento florístico e fitosociológico do extrato arbustivo-arbóreo de dois ambientes na Vila Santa Catarina, Serra do Mel, RN. Revista de Biologia e Ciências da Terra, v. 3, n. $2,2003$. 
Marangon, G. P. et al. Estrutura e padrão espacial da vegetação em uma área de caatinga. Floresta, v. 43, n. 1, p. 83-92, 2013. DOI: 10.5380/rf.v43i1.27807.

Mendes, M. R. A. \& Castro, A. A. J. F. Vascular flora of semi-arid region, São José do Piaui, state of Piaui, Brazil. Check List, v. 6, n. 1, p. 39-44, 2010.

Mendes, M. R. A. Florística e fitossociologia de um fragmento de caatinga arbórea, São José do Piauí, Piauí. 2003. 110 f. Dissertação (Mestrado em Biologia Vegetal) - Universidade Federal de Pernambuco, Recife.

Moreira, A. R. P. et al. Composição florística e análise fitossociológica arbustivo arbóreo no município de Caraúbas-RN. Revista Verde, v. 2, n. 1, p. 113-126, 2007.

Moreira, H. L. (Coord.). Zoneamento geoambiental e agroecológico do Estado de Goiás: região nordeste. Rio de Janeiro: IBGE, 1995. 178 p. (Estudos e pesquisas em geociências, 3).

Murphy, P. G. \& Lugo, A. E. Ecology of tropical dry forest. Annual Review of Ecology and Systematics, v. 17, p. 67-88, 1986. DOI: 10.1146/annurev.es.17.110186.000435.

Nascimento, C. E. S. et al. Phytosociology of the remaining xerophytic woodland associated to an environmental gradient at the banks of the São Francisco river - Petrolina, Pernambuco, Brazil. Revista Brasileira de Botânica, v. 26, n. 3, p. 271-287, 2003. DOI: 10.1590/S0100-84042003000300001.

Oliveira, P. T. B. et al. Florística e fitossociologia de quatro remanescentes vegetacionais em áreas de serra no Cariri Paraibano. Revista Caatinga, v. 22, n. 4, p. 169-178, 2009.

Parente, H. N. et al. Parâmetros fitossociológicos do estrato arbóreoarbustivo em áreas contíguas de caatinga no cariri paraibano. Revista Científica de Produção Animal, v. 12, n. 2, p. 138-141, 2010. DOI: 10.15528/2176-4158/rcpa.v12n2p138-141.

Pereira, I. M. et al. Composição florística e análise fitossociológica do componente arbustivo-arbóreo de um remanescente florestal no agreste paraibano. Acta Botanica Brasilica, v. 16, n. 3, p. 357-369, 2002. DOI: 10.1590/S0102-33062002000300009.

Pereira, I. M. et al. Regeneração natural em um remanescente de Caatinga sob diferentes níveis de perturbação, no agreste paraibano. Acta Botanica Brasilica, v. 15, n. 3, p. 413-426, 2001. DOI: 10.1590/ S0102-33062001000300010.

Pereira Júnior, L. R. et al. Composição florística e fitossociológica de um fragmento de caatinga em Monteiro, PB. Holos, v. 28, n. 6, p. 73-86, 2012. DOI: 10.15628/holos.2012.1188.

Pimentel, D. J. O. Dinâmica da vegetação lenhosa em área de caatinga, Floresta-PE. 2011. 62 f. Dissertação (Mestrado em Ciências Florestais) - Universidade Federal Rural de Pernambuco, Recife.
Pinheiro, K. et al. Floristic composition of different soil types in a semi-arid region of Brazil. Revista Caatinga, v. 23, n. 2, p. 68-77, 2010.

Queiroz, J. A. et al. Análise da estrutura fitossociológica da Serra do Monte, Boqueirão, Paraíba. Revista de Biologia e Ciências da Terra, v. 6, n. 1, p. 251-256, 2006.

Ramos, M. A. Plantas usadas como combustível em uma área de caatinga (Nordeste do Brasil): seleção de espécies, padrões de coleta e qualidade do recurso. 2007. 86 f. Dissertação (Mestrado em Ciências Florestais) - Universidade Federal Rural de Pernambuco, Recife.

Rodal, M. J. N. et al. Composição florística de um trecho de vegetação arbustiva caducifólia, no município de Ibimirim, PE, Brasil. Acta Botanica Brasilica, v. 13, n. 1, p. 15-28, 1999. DOI: 10.1590/S010233061999000100003.

Rodal, M. J. N. et al. Estrutura da vegetação caducifólia espinhosa (Caatinga) de uma área do sertão central de Pernambuco. Hoehnea, v. 35, n. 2, p. 209-217, 2008a. DOI: 10.1590/S223689062008000200004.

Rodal, M. J. N. et al. Levantamento quantitativo das plantas lenhosas em trechos de vegetação de caatinga em Pernambuco. Revista Caatinga, v. 21, n. 3, p. 192-206, 2008 b.

Sampaio, E. V. S. B. \& Gamarra-Rojas, C. F. L. Uso das plantas em Pernambuco. In: Tabarelli M. \& Silva, J. M. C. (Org.). Diagnóstico da biodiversidade de Pernambuco. Recife: Massangana, 2002. p. 633-645.

Santana, J. A. S. \& Souto, J. S. Diversidade e estrutura fitossociológica da caatinga na Estação Ecológica do Seridó-RN. Revista de Biologia e Ciências da Terra, v. 6, n. 2, p. 232-242, 2006.

Santos, R. M. et al. Estrutura e florística de um remanescente florestal na fazenda ribeirão, município de Juvenília, MG, Brasil. Revista Caatinga, v. 21, n. 4, p. 154-162, 2008.

Silva, L. A. \& Scariot, A. Comunidade arbórea de uma floresta estacional decídua sobre afloramento calcário na Bacia do rio Paraná. Revista Árvore, v. 28, n. 1, p. 61-67, 2004. DOI: 10.1590/S010067622004000100008 .

Souza, P. F. Estudos fitossociológicos e dendrométricos em um fragmento de caatinga, São José de Espinharas - PB, Patos. 2012. 97 f. Dissertação (Mestrado em Ciências Florestais) - Universidade Federal de Campina Grande, Patos.

Swaine, M. D. et al. Structure and dynamic of a tropical dry forest in Ghana. Vegetatio, v. 88, n. 1, p. 31-51, 1990. DOI: 10.1007/ BF00032601.

Vieira Júnior, R. L. Caracterização do componente arbustivoarbóreo em um fragmento de Caatinga no Município de Caicó - RN. 2014. 68 f. Dissertação (Mestrado em Ciências Florestais) - Universidade Federal de Campina Grande, Campina Grande. 